\title{
Common Fixed Points For Weak Compatible Maps In Fuzzy Metric Spaces
}

\author{
*Praveen Kumar Sharma, **Sanjay Choudhary and *** Kamal Wadhwa \\ * Department of Mathematics, IES, IPS Academy, Rajendra Nagar, A.B. Road, \\ Indore - 452012 (M.P.), India \\ praveen_jan1980@rediffmail.com \\ **Professor and Head department of Mathematics, Govt. Narmada \\ Mahavidhalaya Hoshangabad (M.P.), India \\ bpi253636@yahoo.in \\ ***Asst. Professor of Mathematics, Govt. Narmada Mahavidhalaya \\ Hoshangabad (M.P.), India \\ wadhwakamal68@gmail.com
}

\begin{abstract}
In this note we shall prove common fixed point theorems for four mappings under weak compatible condition in the setting of fuzzy metric space, fuzzy 2-metric space, and fuzzy 3-metric spaces. Our results generalize the results of Singh, Jain and Jain.
\end{abstract}

Mathematics Subject Classification: 47H10, 54H25

Keywords: Fuzzy metric space; fuzzy 2-metric space ; fuzzy 3-metric space ; t-norm; common fixed points; compatible maps; weak compatible maps.

\section{Introduction}

The concept of fuzzy sets was first given by Zadeh [24] in 1965. Then Kramosil and Michalek [9] introduced the concept of fuzzy metric space. The Fuzzy version of banach contraction principle was given by Grabiec [5] in 1988.This is a mile stone in developing the fixed point theory in fuzzy metric space. 
The improving commutativity in fixed point theorems by using weakly commuting maps in metric spaces was initiated by Sessa [11]. Later on, this method was enlarged to compatible maps by Jungck [7]. The concepts of Rweakly commuting maps and compatible maps in fuzzy metric space were introduced by Vasuki [22] and Mishra et al [10] respectively. The concepts of compatible maps of type $(\alpha)$ and compatible maps of type $(\beta)$ were also introduced by cho [1]. In fact, Jungck and Rhoades [8] termed a pair of self- maps to be coincidentally commuting or equivalently weak compatible if they commute at their coincidence points. The concept of 2-metric space was initiated by Gahler [3] whose abstract properties were suggested by the area function in Euclidian space. Now it is natural to expect the 3-metric space which is suggested by using the volume function.

Many authors have proved different fixed point theorems in fuzzy metric spaces (for example. [6], [18], [23]).

In this paper we shall prove common fixed point theorems for four mappings under weak compatible condition in the setting of fuzzy metric space, fuzzy 2-metric space, and fuzzy 3-metric spaces. Our results generalize the results of Singh, Jain and Jain [18].

\section{Preliminary Notes}

Definition 2.1:- A binary operation $*:[0,1] \times[0,1] \rightarrow[0,1]$ is called a "continuous t-norm" if $([0,1], *)$ is an abelian topological monoid with unit 1 such that- $\mathrm{a}^{*} \mathrm{~b} \leq \mathrm{c}^{*} \mathrm{~d}$ whenever $\mathrm{a} \leq \mathrm{c}$ and $\mathrm{b} \leq \mathrm{d}$ for all $\mathrm{a}, \mathrm{b}, \mathrm{c}$, and $\mathrm{d} \in[0,1]$.

Examples of $\mathrm{t}$-norm are $\mathrm{a} * \mathrm{~b}=\mathrm{ab}$ and $\mathrm{a} * \mathrm{~b}=\min \{\mathrm{a}, \mathrm{b}\}$

Definition 2.2:- The 3- tuple $(X, M, *)$ is called a fuzzy metric space if $X$ is an arbitrary set, $*$ is a continuous t-norm and $\mathrm{M}$ is a fuzzy set in $\mathrm{X}^{2} \times[0, \infty)$ satisfying the following condition's for all $\mathrm{x}, \mathrm{y}, \mathrm{z} \in \mathrm{X}$ and $\mathrm{s}, \mathrm{t}>0$ 
(FM-1) $\quad \mathrm{M}(\mathrm{x}, \mathrm{y}, 0)=0$;

(FM-2) $\quad M(x, y, t)=1$ for all $t>0$ iff $x=y$ :

(FM-3) $\quad \mathrm{M}(\mathrm{x}, \mathrm{y}, \mathrm{t})=\mathrm{M}(\mathrm{y}, \mathrm{x}, \mathrm{t})$;

(FM-4) $\quad \mathrm{M}(\mathrm{x}, \mathrm{y}, \mathrm{t}) * \mathrm{M}(\mathrm{y}, \mathrm{z}, \mathrm{s}) \leq \mathrm{M}(\mathrm{x}, \mathrm{z}, \mathrm{t}+\mathrm{s})$;

$(\mathrm{FM}-5) \quad \mathrm{M}(\mathrm{x}, \mathrm{y},):.[0, \infty) \rightarrow[0,1]$ is left continuous;

(FM-6) $\quad \lim _{t \rightarrow \infty} M(x, y, t)=1$.

Note that $\mathrm{M}(\mathrm{x}, \mathrm{y}, \mathrm{t})$ can be thought of as the degree of nearness between $\mathrm{x}$ and $y$ with respect to $t$. We Identify $x=y$ with $M(x, y, t)=1$ for all $t>0$. The following example shows that every metric space induces a fuzzy metric space.

Example- 2.3:- Let $(X, d)$ be a metric space. Define $a * b=\min \{a, b\}$ and for all $\mathrm{x}, \mathrm{y} \in \mathrm{X}, \mathrm{M}(\mathrm{x}, \mathrm{y}, \mathrm{t})=(\mathrm{t} / \mathrm{t}+\mathrm{d}(\mathrm{x}, \mathrm{y}))$ for all $\mathrm{t}>0$. With $\mathrm{M}(\mathrm{x}, \mathrm{y}, 0)=0$. Then $(X$, $\left.\mathrm{M},{ }^{*}\right)$ is a fuzzy metric space. It is called the fuzzy metric space induced by the metric space $(X, d)$.

Lemma 2.4 ( Grabiec [5]) : For all $x, y \in X, \quad M(x, y,$.$) is a non-decreasing$ function.

Definition 2.5 (Grabiec [5]): Let $\left(\mathrm{X}, \mathrm{M},{ }^{*}\right)$ be a fuzzy metric space. A sequence $\left\{\mathrm{x}_{\mathrm{n}}\right\}$ in $\mathrm{X}$ is said to be convergent to a point $\mathrm{x} \in \mathrm{X}$

If $\lim _{n \rightarrow \infty} M\left(x_{n}, x, t\right)=1$ for all $t>0$. Further, the sequence $\left\{x_{n}\right\}$ is said to be a Cauchy sequence in $X$, if $\lim _{n \rightarrow \infty} M\left(x_{n}, x_{n+p}, t\right)=1$ for all $t>0$ and $p>0$. The space is said to be complete if every Cauchy sequence in $\mathrm{X}$ converges to a point of $X$.

Remark -1:-Since $*$ is continuous, it follows from (FM-4) that the limit of a sequence in a fuzzy metric space is unique, if it exists.

Definition 2.6:-A function $M$ is continuous in fuzzy metric space iff whenever, $\left\{x_{n}\right\} \rightarrow x$ and $\left\{y_{n}\right\} \rightarrow y$, then $\lim _{n \rightarrow \infty} M\left(x_{n}, y_{n}, t\right)=M(x, y, t)$, for each $t>0$.

Lemmas 2.7 (Mishra et al-[10]):- Let $\left(X, M,{ }^{*}\right)$ be a fuzzy metric space. If there exists a number $\mathrm{k} \in(0,1)$ such that for all $\mathrm{x}, \mathrm{y} \in \mathrm{X}$ and $\mathrm{t}>0, \mathrm{M}(\mathrm{x}, \mathrm{y}, \mathrm{kt}) \geq$ $M(x, y, t)$. Then, $x=y$. 
Definition 2.8 (Mishra et al [10]):- Let A and B be mappings from a fuzzy metric space $\left(\mathrm{X}, \mathrm{M},{ }^{*}\right)$ into itself. Then the mappings are said to be compatible, if $\lim _{n \rightarrow \infty} M\left(A B x_{n}, B A x_{n}, t\right)=1$ for all $t>0$, whenever $\left\{x_{n}\right\}$ is a sequence in $X$ such that, $\lim _{\mathrm{n} \rightarrow \infty} \mathrm{Ax}_{\mathrm{n}}=\lim _{\mathrm{n} \rightarrow \infty} \mathrm{Bx}_{\mathrm{n}}=\mathrm{x} \in \mathrm{X}$.

Definition 2.9:- $\quad$ Let $A$ and $B$ be mappings from a fuzzy metric space $\left(X, M,{ }^{*}\right)$ into itself. The mappings are said to be weak compatible if they commute at their coincidence points, i.e. $\mathrm{Ax}=\mathrm{Bx}$ imlies $\mathrm{ABx}=\mathrm{BAx}$.

Definition 2.10:- A binary operation $*:[0,1] \times[0,1] \times[0,1] \rightarrow[0,1]$ is called a "continuous t-norm" if $([0,1], *)$ is an abelian topological monoid with unit 1 such that- $\mathrm{a} * \mathrm{~b} * \mathrm{c} \leq \mathrm{d} * \mathrm{e} * \mathrm{f}$, whenever $\mathrm{a} \leq \mathrm{d}, \mathrm{b} \leq \mathrm{e}$ and $\mathrm{c} \leq \mathrm{f}$, for all a,b,c,d,e, and $\mathrm{f}$ $\in[0,1]$.

Definition 2.11 (Sharma [20]):- The 3- tuple (X, M,*) is called a fuzzy 2- metric space if $\mathrm{X}$ is an arbitrary set, $*$ is a continuous t-norm and $\mathrm{M}$ is a fuzzy set in $\mathrm{X}^{3} \times$ $[0, \infty)$ satisfying the following condition's for all $\mathrm{x}, \mathrm{y}, \mathrm{z}, \mathrm{u} \in \mathrm{X}$ and $\mathrm{t}, \mathrm{t}_{1}, \mathrm{t}_{2}, \mathrm{t}_{3}>0$;

$\left(\mathrm{FM}^{\prime}-1\right) \quad \mathrm{M}(\mathrm{x}, \mathrm{y}, \mathrm{z}, 0)=0$

$\left(\mathrm{FM}^{\prime}-2\right) \quad \mathrm{M}(\mathrm{x}, \mathrm{y}, \mathrm{z}, \mathrm{t})=1$ for all $\mathrm{t}>0$, if and only if atleast two of the three points are equal;

$\left(\mathrm{FM}^{\prime}-3\right) \quad \mathrm{M}(\mathrm{x}, \mathrm{y}, \mathrm{z}, \mathrm{t})=\mathrm{M}(\mathrm{y}, \mathrm{x}, \mathrm{z}, \mathrm{t})=\mathrm{M}(\mathrm{z}, \mathrm{x}, \mathrm{y} \mathrm{t}) ;($ symmetry $)$

$\left(\mathrm{FM}^{\prime}-4\right) \quad \mathrm{M}\left(\mathrm{x}, \mathrm{y}, \mathrm{z}, \mathrm{t}_{1}+\mathrm{t}_{2}+\mathrm{t}_{3}\right) \geq \mathrm{M}\left(\mathrm{x}, \mathrm{y}, \mathrm{u}, \mathrm{t}_{1}\right) * \mathrm{M}\left(\mathrm{x}, \mathrm{u}, \mathrm{z}, \mathrm{t}_{2}\right) *$

$\mathrm{M}\left(\mathrm{u}, \mathrm{y}, \mathrm{z}, \mathrm{t}_{3}\right)$; this corresponds to tetrahedral inequality in

2-metric space.

$\left(\mathrm{FM}^{\prime}-5\right) \quad \mathrm{M}(\mathrm{x}, \mathrm{y}, \mathrm{z},):.[0, \infty) \rightarrow[0,1]$ is left continuous;

(FM' -6) $\quad \lim _{\mathrm{t} \rightarrow \infty} \mathrm{M}(\mathrm{x}, \mathrm{y}, \mathrm{z}, \mathrm{t})=1$.

The function value $\mathrm{M}(\mathrm{x}, \mathrm{y}, \mathrm{z}, \mathrm{t})$ may be interpreted as the probability that the area of triangle is less than $\mathrm{t}$.

Definition 2.12: Let $\left(X, M,{ }^{*}\right)$ be a fuzzy 2-metric space. A sequence $\left\{x_{n}\right\}$ in $X$ is said to be convergent to a point $x \in X$, if $\lim _{n \rightarrow \infty} M\left(x_{n}, x, a, t\right)=1$, for all $t>0$ 
and for all $\mathrm{a} \in \mathrm{X}$. Further, the sequence $\left\{\mathrm{x}_{\mathrm{n}}\right\}$ is said to be a Cauchy sequence in $X$, if $\lim _{n \rightarrow \infty} M\left(x_{n}, x_{n+p}, a, t\right)=1$ for all $t>0, a \in X$ and $p>0$. The space is said to be complete if every Cauchy sequence in $\mathrm{X}$ converges to a point of $\mathrm{X}$.

Definition 2.13:-A function $M$ is continuous in fuzzy 2-metric space iff whenever, $\left\{\mathrm{x}_{\mathrm{n}}\right\} \rightarrow \mathrm{x}$ and $\left\{\mathrm{y}_{\mathrm{n}}\right\} \rightarrow \mathrm{y}$, then $\lim _{\mathrm{n} \rightarrow \infty} \mathrm{M}\left(\mathrm{x}_{\mathrm{n}}, \mathrm{y}_{\mathrm{n}}, \mathrm{a}, \mathrm{t}\right)=\mathrm{M}(\mathrm{x}, \mathrm{y}, \mathrm{a}, \mathrm{t})$, for all $\mathrm{a} \in \mathrm{X}$ and for each $t>0$.

Definition 2.14:- A binary operation $*:[0,1] \times[0,1] \times[0,1] \times[0,1] \rightarrow[0,1]$ is called a "continuous t-norm" if $([0,1], *)$ is an abelian topological monoid with unit 1 such that $a * b * c * d \leq e * f * g * h$, whenever $a \leq e, b \leq f, c \leq g$ and $d$ $\leq \mathrm{h}$, for all a,b,c,d,e, f, g and $\mathrm{h} \in[0,1]$.

Definition 2.15 (Sharma [20]):- The 3- tuple (X, M,*) is called a fuzzy 3- metric space if $\mathrm{X}$ is an arbitrary set, $*$ is a continuous t-norm and $\mathrm{M}$ is a fuzzy set in $\mathrm{X}^{4} \times$ $[0, \infty)$ satisfying the following condition's for all $\mathrm{x}, \mathrm{y}, \mathrm{z}, \mathrm{u}, \mathrm{w} \in \mathrm{X}$ and $\mathrm{t}, \mathrm{t}_{1}, \mathrm{t}_{2}, \mathrm{t}_{3}$, $\mathrm{t}_{4}>0$;

(FM"-1) $\quad \mathrm{M}(\mathrm{x}, \mathrm{y}, \mathrm{z}, \mathrm{w}, 0)=0$;

(FM"-2) $\quad \mathrm{M}(\mathrm{x}, \mathrm{y}, \mathrm{z}, \mathrm{w}, \mathrm{t})=1$ for all $\mathrm{t}>0$, if and only if atleast two of the four points are equal;

$(F M "-3) \quad M(x, y, z, w, t)=M(y, x, z, w, t)=M(w, z, x, y t)=$

(Symmetry)

$\left(\mathrm{FM}\right.$ "-4) $\quad \mathrm{M}\left(\mathrm{x}, \mathrm{y}, \mathrm{z}, \mathrm{t}_{1}+\mathrm{t}_{2}+\mathrm{t}_{3}+\mathrm{t}_{4}\right) \geq \mathrm{M}\left(\mathrm{x}, \mathrm{y}, \mathrm{z}, \mathrm{u}, \mathrm{t}_{1}\right) * \mathrm{M}\left(\mathrm{x}, \mathrm{y}, \mathrm{u}, \mathrm{w}, \mathrm{t}_{2}\right) *$ $\mathrm{M}\left(\mathrm{x}, \mathrm{u}, \mathrm{z}, \mathrm{w}, \mathrm{t}_{3}\right) * \mathrm{M}\left(\mathrm{u}, \mathrm{y}, \mathrm{z}, \mathrm{w}, \mathrm{t}_{4}\right)$

$\left(\mathrm{FM}^{\prime \prime}-5\right) \quad \mathrm{M}(\mathrm{x}, \mathrm{y}, \mathrm{z}, \mathrm{w},):.[0, \infty) \rightarrow[0,1]$ is left continuous; (FM"-6) $\quad \lim _{\mathrm{t} \rightarrow \infty} \mathrm{M}(\mathrm{x}, \mathrm{y}, \mathrm{z}, \mathrm{w}, \mathrm{t})=1$.

Definition 2.16: Let $\left(X, M,{ }^{*}\right)$ be a fuzzy 3-metric space. A sequence $\left\{x_{n}\right\}$ in $X$ is said to be convergent to a point $x \in X$, if $\lim _{n \rightarrow \infty} M\left(x_{n}, x, a, b, t\right)=1$, for all $t>$ 0 and for all $a, b \in X$. Further, the sequence $\left\{x_{n}\right\}$ is said to be a Cauchy sequence in $X$, if $\lim _{n \rightarrow \infty} M\left(x_{n}, x_{n+p}, a, b, t\right)=1$ for all $t>0, a, b \in X$ and $p>0$. The space is said to be complete if every Cauchy sequence in $\mathrm{X}$ converges to a point of $\mathrm{X}$. 
Definition 2.17:-A function $M$ is continuous in fuzzy 3-metric space iff whenever, $\left\{x_{n}\right\} \rightarrow x$ and $\left\{y_{n}\right\} \rightarrow y$, then $\lim _{n \rightarrow \infty} M\left(x_{n}, y_{n}, a, b, t\right)=M(x, y, a, b, t)$, for all a, $\mathrm{b} \in \mathrm{X}$ and for each $\mathrm{t}>0$.

* Lemmas 2.4,

2.7, and Remark 1 holds good for a fuzzy 2-metric space and a fuzzy 3-metric space as well.

Definition 2.18 (Mishra et al [10]):- Let A and B be mappings from a fuzzy 2metric space $\left(\mathrm{X}, \mathrm{M},{ }^{*}\right)$ into itself. Then the mappings are said to be compatible, if $\lim _{n \rightarrow \infty} M\left(A B x_{n}, B A x_{n}, a, t\right)=1$ for all $t>0$, and for all $a \in X$, whenever $\left\{x_{n}\right\}$ is a sequence in $\mathrm{X}$ such that, $\lim _{\mathrm{n} \rightarrow \infty} \mathrm{Ax}_{\mathrm{n}}=\lim _{\mathrm{n} \rightarrow \infty} \mathrm{Bx}_{\mathrm{n}}=\mathrm{x} \in \mathrm{X}$. Let $\mathrm{A}$ and $\mathrm{B}$ be mappings from a fuzzy 3 -metric space $\left(\mathrm{X}, \mathrm{M},{ }^{*}\right)$ into itself. Then the mappings are said to be compatible, if $\lim _{n \rightarrow \infty} \mathrm{M}\left(\mathrm{ABx}_{\mathrm{n}}, \mathrm{BAx}_{\mathrm{n}}, \mathrm{a}, \mathrm{b}, \mathrm{t}\right)=1$ for all $\mathrm{t}>0$, and for all $\mathrm{a}, \mathrm{b} \in \mathrm{X}$, whenever $\left\{\mathrm{x}_{\mathrm{n}}\right\}$ is a sequence in $\mathrm{X}$ such that, $\lim _{\mathrm{n} \rightarrow \infty} \mathrm{Ax}_{\mathrm{n}}=$ $\lim _{\mathrm{n} \rightarrow \infty} \mathrm{Bx}_{\mathrm{n}}=\mathrm{x} \in \mathrm{X}$

Singh, Jain and Jain [18] proved the following theorem for four self maps in complete fuzzy metric space.

Theorem A ([18]):- Let A, B, S and T be self mappings of a complete fuzzy metric space $\left(\mathrm{X}, \mathrm{M},{ }^{*}\right)$. Suppose that they satisfy the following conditions;

(A.11) $\quad \mathrm{A}(\mathrm{X}) \subseteq \mathrm{T}(\mathrm{X}), \mathrm{B}(\mathrm{X}) \subseteq \mathrm{S}(\mathrm{X})$;

(A.12) A or $\mathrm{S}$ is continuous;

(A.13) the pair $(\mathrm{A}, \mathrm{S})$ is compatible and $(\mathrm{B}, \mathrm{T})$ is weak compatible;

(A.14) There exists $\mathrm{k} \in(0,1)$ such that $\forall \mathrm{x}, \mathrm{y} \in \mathrm{X}$ and $\mathrm{t}>0$, $M(A x, B y, k t) \geq \operatorname{Min}\{M(B y, T y, t), M(S x, T y, t), M(A x, S x, t)\}$

(A.15) $\lim _{\mathrm{t} \rightarrow \infty} \mathrm{M}(\mathrm{x}, \mathrm{y}, \mathrm{t})=1$; for all $\mathrm{x}, \mathrm{y} \in \mathrm{X}$ and $\mathrm{t}>0$.

Then A, B, S and T have a unique common fixed point in $\mathrm{X}$. 


\section{Main Results}

We prove the following.

Theorem 3.1:- Let A, B, S and T be self mappings of a complete fuzzy metric space $(\mathrm{X}, \mathrm{M}, *)$. Suppose that they satisfy the following conditions;

$$
\mathrm{A}(\mathrm{X}) \subseteq \mathrm{T}(\mathrm{X}), \mathrm{B}(\mathrm{X}) \subseteq \mathrm{S}(\mathrm{X})
$$

(3.12) the pairs $(\mathrm{A}, \mathrm{S})$ and $(\mathrm{B}, \mathrm{T})$ are weak compatible;

(3.13) There exists $\mathrm{k} \in(0,1)$ such that $\forall \mathrm{x}, \mathrm{y} \in \mathrm{X}$ and $\mathrm{t}>0$, $M(A x, B y, k t) \geq \operatorname{Min}\{M(B y, T y, t), M(S x, T y, t), M(A x, S x, t)\} ;$

Then A, B, S and T have a unique common fixed point in $\mathrm{X}$.

Proof: - Let $\mathrm{x}_{0} \in \mathrm{X}$ be an arbitrary point.

As $\mathrm{A}(\mathrm{X}) \subseteq \mathrm{T}(\mathrm{X})$ and $\mathrm{B}(\mathrm{X}) \subseteq \mathrm{S}(\mathrm{X})$. Then there exists $\mathrm{x}_{1}, \mathrm{x}_{2} \in \mathrm{X}$ such that $\mathrm{Ax}_{0}$

$=\mathrm{Tx}_{1}, \mathrm{Bx}_{1}=\mathrm{Sx}_{2}$.Inductively, we can construct sequences $\left\{\mathrm{y}_{\mathrm{n}}\right\}$ and $\left\{\mathrm{x}_{\mathrm{n}}\right\}$ in $\mathrm{X}$ such that

$$
\mathrm{Y}_{2 \mathrm{n}+1}=\mathrm{Ax}_{2 \mathrm{n}}=\mathrm{Tx}_{2 \mathrm{n}+1}, \mathrm{Y}_{2 \mathrm{n}+2}=\mathrm{Bx}_{2 \mathrm{n}+1}=\mathrm{Sx}_{2 \mathrm{n}+2}, \text { for } \mathrm{n}=0,1,2 \ldots
$$

We first show that $\left\{y_{n}\right\}$ is a Cauchy sequence in $X$.

Now, by (3.13) with $x=x_{2 n}, y=x_{2 n+1}$.

We obtain that,

$$
\begin{aligned}
& \mathrm{M}\left(\mathrm{Ax}_{2 \mathrm{n}}, \mathrm{Bx}_{2 \mathrm{n}+1}, \mathrm{Kt}\right)=\mathrm{M}\left(\mathrm{y}_{2 \mathrm{n}+1}, \mathrm{y}_{2 \mathrm{n}+2}, \mathrm{kt}\right) \\
& \geq \operatorname{Min}\left\{\mathrm{M}\left(\mathrm{Bx}_{2 \mathrm{n}+1}, \mathrm{Tx}_{2 \mathrm{n}+1}, \mathrm{t}\right), \mathrm{M}\left(\mathrm{Sx}_{2 \mathrm{n}}, \mathrm{Tx}_{2 \mathrm{n}+1}, \mathrm{t}\right), \mathrm{M}\left(\mathrm{Ax}_{2 \mathrm{n}}, \mathrm{Sx}_{2 \mathrm{n}}, \mathrm{t}\right)\right\} \\
& \geq \operatorname{Min}\left\{\mathrm{M}\left(\mathrm{y}_{2 \mathrm{n}+1}, \mathrm{y}_{2 \mathrm{n}+2}, \mathrm{t}\right), \mathrm{M}\left(\mathrm{y}_{2 \mathrm{n}}, \mathrm{y}_{2 \mathrm{n}+1}, \mathrm{t}\right), \mathrm{M}\left(\mathrm{y}_{2 \mathrm{n}}, \mathrm{y}_{2 \mathrm{n}+1}, \mathrm{t}\right)\right\} \\
& \geq \operatorname{Min}\left\{\mathrm{M}\left(\mathrm{y}_{2 \mathrm{n}+1}, \mathrm{y}_{2 \mathrm{n}+2}, \mathrm{t}\right), \mathrm{M}\left(\mathrm{y}_{2 \mathrm{n}}, \mathrm{y}_{2 \mathrm{n}+1}, \mathrm{t}\right)\right\} \rightarrow \text { (i) }
\end{aligned}
$$

Thus we have,

$M\left(y_{2 n+1}, y_{2 n+2}, t\right) \geq \operatorname{Min}\left\{M\left(y_{2 n+1}, y_{2 n+2}, t / k\right), M\left(y_{2 n}, y_{2 n+1}, t / k\right) \rightarrow(\right.$ ii $)$

By putting (ii) in (i), we have,

$$
\begin{aligned}
M\left(y_{2 n+1}, y_{2 n+2}, k t\right) & \geq \operatorname{Min}\left\{M\left(y_{2 n+1}, y_{2 n+2}, t / k\right), M\left(y_{2 n}, y_{2 n+1}, t / k\right), M\left(y_{2 n}, y_{2 n+1}, t\right)\right\} \\
= & \operatorname{Min}\left\{M\left(y_{2 n+1}, y_{2 n+2}, t / k\right), M\left(y_{2 n}, y_{2 n+1}, t\right)\right\} \\
\geq & \operatorname{Min}\left\{M\left(y_{2 n+1}, y_{2 n+2}, t / k^{2}\right), M\left(y_{2 n}, y_{2 n+1}, t / k^{2}\right), M\left(y_{2 n}, y_{2 n+1}, t\right)\right\} \\
= & M i n\left\{M\left(y_{2 n+1}, y_{2 n+2}, t / k^{2}\right), M\left(y_{2 n}, y_{2 n+1}, t\right)\right\}
\end{aligned}
$$




$$
\begin{aligned}
& \geq \ldots \ldots \ldots \\
& \geq \operatorname{Min}\left\{M\left(y_{2 n+1}, y_{2 n+2}, t / k^{m}\right), M\left(y_{2 n}, y_{2 n+1}, t\right)\right\}
\end{aligned}
$$

Taking limit as $\mathrm{m} \rightarrow \infty$, we have,

$$
\mathrm{M}\left(\mathrm{y}_{2 \mathrm{n}+1}, \mathrm{y}_{2 \mathrm{n}+2}, \mathrm{kt}\right) \geq \mathrm{M}\left(\mathrm{y}_{2 \mathrm{n}}, \mathrm{y}_{2 \mathrm{n}+1}, \mathrm{t}\right), \forall \mathrm{t}>0
$$

Similarly, we also have

$$
\mathrm{M}\left(\mathrm{y}_{2 \mathrm{n}+2}, \mathrm{y}_{2 \mathrm{n}+3}, \mathrm{kt}\right) \geq \mathrm{M}\left(\mathrm{y}_{2 \mathrm{n}+2}, \mathrm{y}_{2 \mathrm{n}+1}, \mathrm{t}\right) \forall \mathrm{t}>0
$$

Thus, for all $\mathrm{n}$, and $\mathrm{t}>0$.

$$
\begin{aligned}
& M\left(y_{n}, y_{n+1}, k t\right) \geq M\left(y_{n}, y_{n-1}, t\right) \text { Therefore, } \\
& M\left(y_{n}, y_{n+1}, t\right) \geq M\left(y_{n-1}, y_{n}, t / k\right) \geq M\left(y_{n-2}, y_{n-1}, t / k^{2}\right) \geq \ldots \ldots . . M\left(y_{0}, y_{1}, t / k^{n}\right)
\end{aligned}
$$

Hence, $\lim _{\mathrm{n} \rightarrow \infty} \mathrm{M}\left(\mathrm{y}_{\mathrm{n}}, \mathrm{y}_{\mathrm{n}+1}, \mathrm{t}\right)=1 . \forall \mathrm{t}>0$

Now, for any integer $\mathrm{p}$, we have

$$
M\left(y_{n}, y_{n+p}, t\right) \geq M\left(y_{n}, y_{n+1}, t / p\right) * M\left(y_{n+1}, y_{n+2}, t / p\right) * \ldots . . \ldots . . * M\left(y_{n+p-1}, y_{n+p}, t / p\right)
$$

Therefore, $\lim _{n \rightarrow \infty} M\left(y_{n}, y_{n+p}, t\right)=1 * 1 * 1 * \ldots \ldots * 1=1$

$$
\lim _{n \rightarrow \infty} M\left(y_{n}, y_{n+p}, t\right)=1 \text {. }
$$

This shows that $\left\{\mathrm{y}_{\mathrm{n}}\right\}$ is a Cauchy sequence in $\mathrm{X}$, which is complete.

Therefore, $\left\{\mathrm{y}_{\mathrm{n}}\right\}$ converges to $\mathrm{z} \in \mathrm{X}$.

We have the following subsequences;

$$
\begin{aligned}
\left\{\mathrm{Ax}_{2 \mathrm{n}}\right\} \rightarrow \mathrm{z}, & \left\{\mathrm{Bx}_{2 \mathrm{n}+1}\right\} \rightarrow \mathrm{z} \rightarrow(1) \\
\left\{\mathrm{Sx}_{2 \mathrm{n}}\right\} \rightarrow \mathrm{z}, & \left\{\mathrm{Tx}_{2 \mathrm{n}+1}\right\} \rightarrow \mathrm{z} \rightarrow(2)
\end{aligned}
$$

Since $A(X) \subseteq T(X) \quad \exists$ for $p \in X$ such that $p=T^{-1} z$ i.e. $T p=z$.

By (3.13) we have. (at $\left.x=x_{2 n}, y=p\right)$

$M\left(\mathrm{Ax}_{2 \mathrm{n}}, \mathrm{Bp}, \mathrm{kt}\right) \geq \operatorname{Min}\left\{\mathrm{M}(\mathrm{Bp}, \mathrm{Tp}, \mathrm{t}), \mathrm{M}\left(\mathrm{Sx}_{2 \mathrm{n}}, \mathrm{Tp}, \mathrm{t}\right) \mathrm{M}\left(\mathrm{Ax}_{2 \mathrm{n}}, \mathrm{Sx}_{2 \mathrm{n}}, \mathrm{t}\right)\right\}$

$\mathrm{M}\left(\mathrm{Ax}_{2 \mathrm{n}}, \mathrm{Bp}, \mathrm{kt}\right) \geq \operatorname{Min}\left\{\mathrm{M}(\mathrm{Bp}, \mathrm{z}, \mathrm{t}), \mathrm{M}\left(\mathrm{Sx}_{2 \mathrm{n}}, \mathrm{z}, \mathrm{t}\right), \mathrm{M}\left(\mathrm{Ax}_{2 \mathrm{n}}, \mathrm{Sx}_{2 \mathrm{n}}, \mathrm{t}\right\}\right.$

Taking the limit $\mathrm{n} \rightarrow \infty$ and using (i) and (ii) we have,

$\mathrm{M}(\mathrm{z}, \mathrm{Bp}, \mathrm{kt}) \geq \operatorname{Min}\{\mathrm{M}(\mathrm{Bp}, \mathrm{z}, \mathrm{t}), \mathrm{M}(\mathrm{z}, \mathrm{z}, \mathrm{t}), \mathrm{M}(\mathrm{z}, \mathrm{z}, \mathrm{t})\}$

$\mathrm{M}(\mathrm{z}, \mathrm{Bp}, \mathrm{kt}) \geq \mathrm{M}(\mathrm{Bp}, \mathrm{z}, \mathrm{t})$

Therefore by lemma (2.7) we have.

$\mathrm{z}=\mathrm{Bp}$. Since $\mathrm{z}=\mathrm{Tp}$ therefore $\mathrm{z}=\mathrm{Bp}=\mathrm{Tp}$.

i.e. $\mathrm{p}$ is a coincidence point of $\mathrm{B}$ and $\mathrm{T}$. 
Similarly, since $\mathrm{B}(\mathrm{X}) \subseteq \mathrm{S}(\mathrm{X}) ; \exists \mathrm{q} \in \mathrm{X}$ such that $\mathrm{q}=\mathrm{S}^{-1} \mathrm{z}$ i.e $\mathrm{Sq}=\mathrm{z}$.

By (3.13) we have (at $x=q, y=x_{2 n+1}$ )

$M\left(A q, B x_{2 n+1}, k t\right) \geq \operatorname{Min}\left\{M\left(B x_{2 n+1}, T x_{2 n+1}, t\right), M\left(S q, T x_{2 n+1}, t\right), M(A q, S q, t)\right\}$.

$M\left(A q, B x_{2 n+1}, k t\right) \geq \operatorname{Min}\left\{M\left(B x_{2 n+1}, T x_{2 n+1}, t\right), M\left(z, T_{2 n+1}, t\right), M(A q, z, t)\right\}$.

Taking the limit $\mathrm{n} \rightarrow \infty$ and using (i) and (ii) we have;

$M(A q, z, k t) \geq \operatorname{Min}\{M(z, z, t), M(z, z, t), M(z, z, t), M(A q, z, t)\}$.

$\mathrm{M}(\mathrm{Aq}, \mathrm{z}, \mathrm{kt}) \geq \mathrm{M}(\mathrm{Aq}, \mathrm{z}, \mathrm{t})$

Therefore by lemma (2.7) we have.

$\mathrm{Aq}=\mathrm{z}$. Since $\mathrm{Sq}=\mathrm{z}$, therefore, $\mathrm{z}=\mathrm{Aq}=\mathrm{Sq}$. i.e. $\mathrm{q}$ is a coincidence point of $\mathrm{A}$ and $\mathrm{S}$.

Since $\{A, S\}$ is weakly compatible. Therefore by definition of weak compatible we have,

$\mathrm{ASq}=\mathrm{SAq}$ or $\mathrm{Az}=\mathrm{Sz}$.

Similarly $\{B, T\}$ is weakly compatible, therefore by definition of weak compatible we have.

$\mathrm{BTp}=\mathrm{TBp}$ or $\mathrm{Bz}=\mathrm{Tz}$.

Now By (3.13) we have: (at $\mathrm{x}=\mathrm{z}, \mathrm{y}=\mathrm{x}_{2 \mathrm{n}+1}$ )

$M\left(A z, B x_{2 n+1}, k t\right) \geq \operatorname{Min}\left\{M\left(B x_{2 n+1}, T x_{2 n+1}, t\right), M\left(S z, T x_{2 n+1}, t\right), M(A z, S z, t)\right\}$.

$M\left(A z, B x_{2 n+1}, k t\right) \geq \operatorname{Min}\left\{M\left(B x_{2 n+1}, T x_{2 n+1}, t\right), M\left(A z, T x_{2 n+1}, t\right), M(A z, S z, t)\right\}$.

Taking the limit $\mathrm{n} \rightarrow \infty$, we have,

$\mathrm{M}(\mathrm{Az}, \mathrm{z}, \mathrm{kt}) \geq \operatorname{Min}\{\mathrm{M}(\mathrm{z}, \mathrm{z}, \mathrm{t}), \mathrm{M}(\mathrm{Az}, \mathrm{z}, \mathrm{t}), 1\}$.

$\mathrm{M}(\mathrm{Az}, \mathrm{z}, \mathrm{kt}) \geq \mathrm{M}(\mathrm{Az}, \mathrm{z}, \mathrm{t})$

Therefore by lemma (2.7), we have,

$\mathrm{Az}=\mathrm{z}$. Since $\mathrm{Az}=\mathrm{Sz}$, therefore $\mathrm{z}=\mathrm{Az}=\mathrm{Sz}$.

Again by (3.13) we have; (at $\mathrm{x}=\mathrm{x}_{2 \mathrm{n}}, \mathrm{y}=\mathrm{z}$ )

$M\left(A x_{2 n}, B z, k t\right) \geq \operatorname{Min}\left\{M(B z, T z, t), M\left(S x_{2 n}, T z, t\right), M\left(A x_{2 n}, S x_{2 n}, t\right)\right\}$

$\mathrm{M}\left(\mathrm{Ax}_{2 \mathrm{n}}, \mathrm{Bz}, \mathrm{kt}\right) \geq \operatorname{Min}\left\{\mathrm{M}(\mathrm{Bz}, \mathrm{Bz}, \mathrm{t}), \mathrm{M}\left(\mathrm{Sx}_{2 \mathrm{n}}, \mathrm{Bz}, \mathrm{t}\right), \mathrm{M}\left(\mathrm{Ax}_{2 \mathrm{n}}, \mathrm{Sx}_{2 \mathrm{n}}, \mathrm{t}\right)\right\}$.

Taking the limit $\mathrm{n} \rightarrow \infty$ we have;

$M(z, B z, k t) \geq \operatorname{Min}\{1, M(z, B z, t), M(z, z, t)\}$ 
$\mathrm{M}(\mathrm{z}, \mathrm{Bz}, \mathrm{kt}) \geq \operatorname{Min}\{1, \mathrm{M}(\mathrm{z}, \mathrm{Bz}, \mathrm{t}), 1\}$

$\mathrm{M}(\mathrm{z}, \mathrm{Bz}, \mathrm{kt}) \geq \mathrm{M}(\mathrm{z}, \mathrm{Bz}, \mathrm{t})$

Therefore by lemma (2.7) we have;

$$
\mathrm{z}=\mathrm{Bz} \text {. Since } \mathrm{Bz}=\mathrm{Tz} \text {, therefore } \mathrm{z}=\mathrm{Bz}=\mathrm{Tz} \text {. }
$$

Thus we have, $\mathrm{z}=\mathrm{Az}=\mathrm{Sz}=\mathrm{Bz}=\mathrm{Tz}$.

Hence $\mathrm{z}$ is a common fixed point of $\mathrm{A}, \mathrm{B}, \mathrm{S}$ and $\mathrm{T}$.

Uniqueness- let $\mathrm{z}$ and $\mathrm{z}$ ' be two common fixed points of the maps $\mathrm{A}, \mathrm{B}, \mathrm{S}$ and $\mathrm{T}$. then

$\mathrm{z}=\mathrm{Az}=\mathrm{Sz}=\mathrm{Bz}=\mathrm{Tz}$ and $\mathrm{z}^{\prime}=\mathrm{Az} \mathrm{z}^{\prime}=\mathrm{Sz} \mathrm{z}^{\prime}=\mathrm{Bz}^{\prime}=\mathrm{Tz} \mathrm{z}^{\prime}$

Now: By (3.13) we have (at $\mathrm{x}=\mathrm{z}, \mathrm{y}=\mathrm{z}^{\prime}$ )

$\mathrm{M}\left(\mathrm{Az}, \mathrm{Bz} z^{\prime}, \mathrm{kt}\right) \geq \operatorname{Min}\left\{\mathrm{M}\left(\mathrm{Bz}^{\prime}, \mathrm{Tz}, \mathrm{t}\right), \mathrm{M}(\mathrm{Sz}, \mathrm{Tz}, \mathrm{t}), \mathrm{M}(\mathrm{Az}, \mathrm{Sz}, \mathrm{t})\right\}$

$M\left(z, z^{\prime}, k t\right) \geq \operatorname{Min}\left\{M\left(z^{\prime}, z^{\prime}, t\right), M\left(z, z^{\prime}, t\right), M(z, z, t)\right\}$

$\mathrm{M}\left(\mathrm{z}, \mathrm{z}^{\prime}, \mathrm{kt}\right) \geq \operatorname{Min}\left\{1, \mathrm{M}\left(\mathrm{z}, \mathrm{z}^{\prime}, \mathrm{t}\right), 1\right\}$

$\left.\mathrm{M}\left(\mathrm{z}, \mathrm{z}^{\prime}, \mathrm{kt}\right) \geq \mathrm{M}\left(\mathrm{z}, \mathrm{z}^{\prime}, \mathrm{t}\right)\right\}$

Therefore by lemma (2.7) we have, $z=z$ '

Hence $\mathrm{z}$ is the unique common fixed point of the four self maps $\mathrm{A}, \mathrm{B}, \mathrm{S}$ and $\mathrm{T}$.

This completes the proof.

If we take $\mathrm{B}=\mathrm{A}$ in theorem 3.1 , we get the following corollary for three self maps.

Corollary 3.2- Let A, S and T be self mappings of a complete fuzzy metric space $(\mathrm{X}, \mathrm{M}, *)$; satisfying;

(3.21) $\mathrm{A}(\mathrm{X}) \subseteq \mathrm{S}(\mathrm{X}) \cap \mathrm{T}(\mathrm{X})$;

(3.22) Pairs (A, S) and (A, T) are weak compatible,

(3.23) M (Ax, Ay, kt) $\geq \operatorname{Min}(M(A y, T y, t), M(S x, T y, t), M(A x, S x, t)\}$

for all $\mathrm{x}, \mathrm{y} \in \mathrm{X}, \mathrm{t}>0$ and $0<\mathrm{k}<1$.

Then, A, S and T have a unique common fixed point in $\mathrm{X}$.

Proof. - The proof is similar to the proof of theorem (3.1). 
If we take $\mathrm{S}=\mathrm{T}=\mathrm{I}$, the identity maps on $\mathrm{X}$ in corollary 3.2 , then the conditions (3.21) and (3.22) are trivially satisfied.

Now, taking only one factor in R.H.S. of the contraction (3.33), we obtain the following corollary as follows;

Corollary 3.3:- Let A be a self map on a complete fuzzy metric space (X, M, *) such that for some $\mathrm{k} \in(0,1)$.

$M(A x, A y, k t) \geq M(x, y, t)$ for all $x, y \in X, t>0$.

Then, A has a unique common fixed point in $\mathrm{X}$.

The following theorem for four self maps in complete fuzzy 2 - metric space was proved by Singh Jain and Jain in [18]

Theorem B [18]. Let A, B, S and T be self mappings of a complete fuzzy 2metric space $(\mathrm{X}, \mathrm{M}, *)$. Suppose that they satisfy the following conditions;

(B.11) $\mathrm{A}(\mathrm{X}) \subseteq \mathrm{T}(\mathrm{X}), \mathrm{B}(\mathrm{X}) \subseteq \mathrm{S}(\mathrm{X})$;

(B.12) A or $\mathrm{S}$ is continuous;

(B.13) The pair (A, S) is compatible and (B, T) is weak compatible.

(B.14) there exists $\mathrm{k} \in(0,1)$ such that for all $\mathrm{x}, \mathrm{y}, \mathrm{a} \in \mathrm{X}$, and $\mathrm{t}>0$, $M(A x, B y, a, k t) \geq \operatorname{Min}\{M(B y, T y, a, t), M(S x, T y, a, t), M(A x, S x, a, t)\}$; (B.15) $\lim _{\mathrm{t} \rightarrow \infty} \mathrm{M}(\mathrm{x}, \mathrm{y}, \mathrm{a}, \mathrm{t})=1$ for all $\mathrm{x}, \mathrm{y}, \mathrm{a} \in \mathrm{X}$ and $\mathrm{t}>0$.

Then A, B, S and T have a unique common fixed point in $\mathrm{X}$.

Now we prove the following;

Theorem 3.4. Let A, B, S and $\mathrm{T}$ be self mappings of a complete fuzzy 2-metric space $(\mathrm{X}, \mathrm{M}, *)$. Suppose that they satisfy the following conditions;

(3.41) $\mathrm{A}(\mathrm{X}) \subseteq \mathrm{T}(\mathrm{X}), \mathrm{B}(\mathrm{X}) \subseteq \mathrm{S}(\mathrm{X})$;

(3.42) the pairs (A, S) and (B, T) are weak compatible;

(3.43) there exists $\mathrm{k} \in(0,1)$ such that for all $\mathrm{x}, \mathrm{y}, \mathrm{a} \in \mathrm{X}$ and $\mathrm{t}>0$;

$M(A x, B y, a, k t) \geq \operatorname{Min}\{M(B y, T y, a, t), M(S x, T y, a, t), M(A x, S x, a, t)\} ;$

Then A, B, S and T have a unique common fixed point in X. 
Proof: As in proof of theorem 3.1, we define the sequences $\left(\mathrm{y}_{\mathrm{n}}\right)$ and $\left(\mathrm{x}_{\mathrm{n}}\right)$ in $\mathrm{X}$ as follows;

$\mathrm{y}_{2 \mathrm{n}+1}=\mathrm{Ax}_{2 \mathrm{n}}=\mathrm{Tx}_{2 \mathrm{n}+1}, \mathrm{y}_{2 \mathrm{n}+2}=\mathrm{Bx}_{2 \mathrm{n}+1}=\mathrm{Sx}_{2 \mathrm{n}+2}, \mathrm{n}=0,1,2 \ldots$

Now, we show that $\left\{y_{n}\right\}$ is a Cauchy sequence in $X$.

By using (3.43) with $\mathrm{x}=\mathrm{x}_{2 \mathrm{n}}, \mathrm{y}=\mathrm{x}_{2 \mathrm{n}+1}$ we obtain that;

$$
\begin{aligned}
& M\left(A_{2 n}, B x_{2 n+1}, a, k t\right)=M\left(y_{2 n+1}, y_{2 n+2}, a, k t\right) \geq \operatorname{Min}\left\{M\left(B x_{2 n+1}, T_{2 n+1}, a, t\right), M\right. \\
& \left.\left(\operatorname{Sx}_{2 n}, T_{2 n+1}, a, t\right), M\left(A x_{2 n}, S x_{2 n}, a, t\right)\right\} \\
& =\operatorname{Min}\left\{M\left(y_{2 n+1}, y_{2 n+2}, a, t\right), M\left(y_{2 n}, y_{2 n+2}, a, t\right), M\left(y_{2 n}, y_{2 n+1}, a, t\right)\right\} \\
& =\operatorname{Min}\left\{M\left(y_{2 n+1}, y_{2 n+2}, a, t\right), M\left(y_{2 n}, y_{2 n+1}, a, t\right)\right\} \ldots \text { (iii) }
\end{aligned}
$$

Thus we have:

$\mathrm{M}\left(\mathrm{y}_{2 \mathrm{n}+1}, \mathrm{y}_{2 \mathrm{n}+2}, \mathrm{a}, \mathrm{t}\right)$

$\geq \operatorname{Min}\left\{\mathrm{M}\left(\mathrm{y}_{2 \mathrm{n}+1}, \mathrm{y}_{2 \mathrm{n}+2}, \mathrm{a}, \mathrm{t} / \mathrm{k}\right) \mathrm{M}\left(\mathrm{y}_{2 \mathrm{n}}, \mathrm{y}_{2 \mathrm{n}+1}, \mathrm{a}, \mathrm{t} / \mathrm{k}\right)\right\}$

By putting (iv) in (iii), we have;

$\mathrm{M}\left(\mathrm{y}_{2 \mathrm{n}+1}, \mathrm{y}_{2 \mathrm{n}+2}, \mathrm{a}, \mathrm{kt}\right)$

$\geq \operatorname{Min}\left\{\mathrm{M}\left(\mathrm{y}_{2 \mathrm{n}+1}, \mathrm{y}_{2 \mathrm{n}+2}, \mathrm{a}, \mathrm{t} / \mathrm{k}\right), \mathrm{M}\left(\mathrm{y}_{2 \mathrm{n}}, \mathrm{y}_{2 \mathrm{n}+1}, \mathrm{a}, \mathrm{t} / \mathrm{k}\right), \mathrm{M}\left(\mathrm{y}_{2 \mathrm{n}}, \mathrm{y}_{2 \mathrm{n}+1}, \mathrm{a}, \mathrm{t}\right)\right\}$

$=\operatorname{Min}\left\{\mathrm{M}\left(\mathrm{y}_{2 \mathrm{n}+1}, \mathrm{y}_{2 \mathrm{n}+2}, \mathrm{a}, \mathrm{t} / \mathrm{k}\right), \mathrm{M}\left(\mathrm{y}_{2 \mathrm{n}}, \mathrm{y}_{2 \mathrm{n}+1}, \mathrm{a}, \mathrm{t}\right)\right\}$

$\geq \operatorname{Min}\left\{M\left(y_{2 n+1}, y_{2 n+2}, a, t / k^{2}\right), M\left(y_{2 n}, y_{2 n+1}, a, t / k^{2}\right), M\left(y_{2 n}, y_{2 n+1}, a, t\right)\right\}$.

$=\operatorname{Min}\left\{M\left(y_{2 n+1}, y_{2 n+2}, a, t / k^{2}\right), M\left(y_{2 n}, y_{2 n+1}, a, t\right)\right\}$

z............................

$\geq \operatorname{Min}\left\{\mathrm{M}\left(\mathrm{y}_{2 \mathrm{n}+1}, \mathrm{y}_{2 \mathrm{n}+2}, \mathrm{a}, \mathrm{t} / \mathrm{k}^{\mathrm{n}}\right), \mathrm{M}\left(\mathrm{y}_{2 \mathrm{n}}, \mathrm{y}_{2 \mathrm{n}+1}, \mathrm{a}, \mathrm{t}\right)\right\}$.

Taking limit as $\mathrm{n} \rightarrow \infty$, we obtain that,

$\mathrm{M}\left(\mathrm{y}_{2 \mathrm{n}+1}, \mathrm{y}_{2 \mathrm{n}+2}, \mathrm{a}, \mathrm{kt}\right) \geq \mathrm{M}\left(\mathrm{y}_{2 \mathrm{n}}, \mathrm{y}_{2 \mathrm{n}+1}, \mathrm{a}, \mathrm{t}\right), \forall \mathrm{t}>0$.

Similarly, we also obtain that,

$M\left(y_{2 n+2}, y_{2 n+3}, a, k t\right) \geq M\left(y_{2 n+2}, y_{2 n+1}, a, t\right), \forall t>0$.

Thus for all $\mathrm{n}$ and $\mathrm{t}>0$, we have

$M\left(y_{n}, y_{n+1}, a, k t\right) \geq M\left(y_{n-1}, y_{n}, a, t\right)$.

Therefore,

$M\left(y_{n}, y_{n+1}, a, t\right) \geq M\left(y_{n-1}, y_{n}, a, t / k\right)$

$\geq \mathrm{M}\left(\mathrm{y}_{\mathrm{n}-2}, \mathrm{y}_{\mathrm{n}-1}, \mathrm{a}, \mathrm{t} / \mathrm{k}^{2}\right)$

$\geq$ 
$\geq \mathrm{M}\left(\mathrm{y}_{0}, \mathrm{y}_{1}, \mathrm{a}, \mathrm{t} / \mathrm{k}^{\mathrm{n}}\right)$

Hence, $\lim _{\mathrm{n} \rightarrow \infty} \mathrm{M}\left(\mathrm{y}_{\mathrm{n}}, \mathrm{y}_{\mathrm{n}+1}, \mathrm{a}, \mathrm{t}\right)=1, \forall \mathrm{t}>0$.

Now, by induction on any integer $\mathrm{p}$, we prove that

$\lim _{\mathrm{n} \rightarrow \infty} \mathrm{M}\left(\mathrm{y}_{\mathrm{n}}, \mathrm{y}_{\mathrm{n}+\mathrm{p}}, \mathrm{a}, \mathrm{t}\right)=1, \forall \mathrm{t}>0$.

Clearly $(V)$ is true for $p=1$. Suppose that $(V)$ is true for $p=m$. i.e $\lim _{\mathrm{n} \rightarrow \infty} \mathrm{M}\left(\mathrm{y}_{\mathrm{n}}, \mathrm{y}_{\mathrm{n}+\mathrm{m}}, \mathrm{a}, \mathrm{t}\right)=1, \forall \mathrm{t}>0$.

Then by using (FM'- 4) we have,

$M\left(y_{n}, y_{n+m+1}, a, t\right)=M\left(y_{n}, y_{n+m}, a, t / 3\right) * . M\left(y_{n}, y_{n+m}, y_{n+m+1}, t / 3\right) *$

$M\left(y_{n+m}, y_{n+m+1}, a, t / 3\right)$.

Therefore we have:

$\lim _{\mathrm{n} \rightarrow \infty} \mathrm{M}\left(\mathrm{y}_{\mathrm{n}}, \mathrm{y}_{\mathrm{n}+\mathrm{m}+1}, \mathrm{a}, \mathrm{t}\right)=1 * 1 * 1=1$.

Hence (V) is true for $\mathrm{p}=\mathrm{m}+1$ Thus (v) holds for all $\mathrm{p}$ and we get $\left\{\mathrm{y}_{\mathrm{n}}\right\}$ is a Cauchy sequence in $X$, which is complete. Therefore $\left\{y_{n}\right\}$ converges to $z \in X$. We have the following subsequences;

$$
\begin{aligned}
& \left\{\mathrm{Ax}_{2 \mathrm{n}}\right\} \rightarrow \mathrm{z},\left\{\mathrm{Bx}_{2 \mathrm{n}+1}\right\} \rightarrow \mathrm{z} . \ldots \\
& \left\{\mathrm{Sx}_{2 \mathrm{n}}\right\} \rightarrow \mathrm{z},\left\{\mathrm{Tx}_{2 \mathrm{n}+1}\right\} \rightarrow \mathrm{z} . \ldots .
\end{aligned}
$$

Since $\mathrm{A}(X) \subseteq \mathrm{T}(X) \quad \exists \mathrm{p} \in \mathrm{X}$ such that $\mathrm{p}=\mathrm{T}^{-1} \mathrm{z}$ i.e. $\mathrm{Tp}=\mathrm{z}$.

By (3.43) we have (at $\left.x=x_{2 n}, y=p\right)$

$M\left(A x_{2 n}, B p, a, k t\right) \geq \operatorname{Min}\left\{M(B p, T p, a, t), M\left(S x_{2 n}, T p, a, t\right), M\left(A_{2 n}, S x_{2 n}, a, t\right)\right\}$

$M\left(A x_{2 n}, B p, a, k t\right) \geq \operatorname{Min}\left\{M(B p, z, a, t), M\left(S_{2 n}, z, a, t\right), M\left(A_{2 n}, S x_{2 n}, a, t\right)\right\}$

Taking the limit $n \rightarrow \infty$ and using (3) and (4) we have;

$M(z, B p, a k t) \geq \operatorname{Min}\{M(B p, z, a, t), M(z, z, a, t), M(z, z, a, t)\}$.

$\mathrm{M}(\mathrm{z}, \mathrm{Bp}, \mathrm{akt}) \geq \mathrm{M}(\mathrm{Bp}, \mathrm{z}, \mathrm{a}, \mathrm{t}), \quad \forall \mathrm{t}>0$

Now; by lemma (2.7) we obtain that $\mathrm{Bp}=\mathrm{z}$.

Since $\mathrm{Tp}=\mathrm{z}$, therefore $\mathrm{Bp}=\mathrm{Tp}=$ z. i.e. $\mathrm{p}$ is a coincidence point of $\mathrm{B}$ and $\mathrm{T}$.

Similarly; since $B(X) \subseteq S(X), \exists q \in X$ such that $q=S^{-1} z$ i.e. $S q=z$.

By (3.43) we have (at $\left.x=q, y=x_{2 n+1}\right)$

$M\left(A q, B x_{2 n+1}, a, k t\right) \geq \operatorname{Min}\left\{M\left(B x_{2 n+1}, T x_{2 n+1}, a, t\right), M\left(S q, \mathrm{Tx}_{2 n+1}, a, t\right), M(A q\right.$,

Sq, a, t) $\}$ 
$M\left(A q, B x_{2 n+1}, a, k t\right) \geq \operatorname{Min}\left\{M\left(B x_{2 n+1}, T x_{2 n+1}, a, t\right), M\left(z, T x_{2 n+1}, a, t\right), M(A q, z\right.$, $\mathrm{a}, \mathrm{t})\}$

Taking the limit $n \rightarrow \infty$ and using (3) and (4) we have;

$M(A q, z, a, k t) \geq \operatorname{Min}\{M(z, z, a, t), M(z, z, a, t), M(A q, z, a, t)\}$

$M(A q, z, a, k t) \geq M(A q, z, a, t)$

Now; by lemma (2.7) we obtain that $\mathrm{Aq}=\mathrm{z}$.

Since $\mathrm{Sq}=\mathrm{z}$, therefore $\mathrm{Aq}=\mathrm{Sq}=\mathrm{z}$. i.e. $\mathrm{q}$ is a coincidence point of $\mathrm{A}$ and $\mathrm{S}$.

Since $\{A, S\}$ is weakly compatible therefore by definition of weak compatible we have;

$\mathrm{ASq}=\mathrm{SAq}$ or $\mathrm{Az}=\mathrm{Sz}$.

Now; by (3.43) we have; (at $\mathrm{x}=\mathrm{z}, \mathrm{y}=\mathrm{x}_{2 \mathrm{n}+1}$ )

$M\left(A z, B x_{2 n+1}, a, k t\right) \geq \operatorname{Min}\left\{M\left(B x_{2 n+1}, T x_{2 n+1}, a, t\right), M\left(S z, T x_{2 n+1}, a, t\right), M(A z\right.$,

$\mathrm{Sz}, \mathrm{a}, \mathrm{t})\}$

$M\left(A z, B x_{2 n+1}, a, k t\right) \geq \operatorname{Min}\left\{M\left(B x_{2 n+1}, T x_{2 n+1}, a, t\right), M\left(A z, \mathrm{Tx}_{2 n+1}, a, t\right), M(A z\right.$, Az, a, t) $\}$

Taking the limit $\mathrm{n} \rightarrow \infty$ and using (3) and (4) we have;

$\mathrm{M}(\mathrm{Az}, \mathrm{z}, \mathrm{a}, \mathrm{kt}) \geq \operatorname{Min}\{\mathrm{M}(\mathrm{z}, \mathrm{z}, \mathrm{a}, \mathrm{t}), \mathrm{M}(\mathrm{Az}, \mathrm{z}, \mathrm{a}, \mathrm{t}), 1\}$

$\mathrm{M}(\mathrm{Az}, \mathrm{z}, \mathrm{a}, \mathrm{kt}) \geq \mathrm{M}(\mathrm{Az}, \mathrm{z}, \mathrm{a}, \mathrm{t})$

Now; by lemma (2.7) we obtain that $\mathrm{Az}=\mathrm{z}$.

Since $\mathrm{Az}=\mathrm{Sz}$ therefore $\mathrm{z}=\mathrm{Az}=\mathrm{Sz}$.

Since $\{B, T\}$ is weakly compatible, therefore by definition of weak compatible we have;

$\mathrm{BTp}=\mathrm{TBp}$ or $\mathrm{Bz}=\mathrm{Tz}$.

Now, by (3.43) we hve: (at $\mathrm{x}=\mathrm{x}_{2 \mathrm{n}}, \mathrm{y}=\mathrm{z}$ )

$M\left(A x_{2 n}, B z, a, k t\right) \geq \operatorname{Min}\left\{M(B z, T z, a, t), M\left(S x_{2 n}, T z, a, t\right), M\left(A_{2 n}, S x_{2 n}, a, t\right)\right\}$

$\mathrm{M}\left(\mathrm{Ax}_{2 \mathrm{n}}, \mathrm{Bz}, \mathrm{a}, \mathrm{kt}\right) \geq \operatorname{Min}\left\{\mathrm{M}(\mathrm{Bz}, \mathrm{Bz}, \mathrm{a}, \mathrm{t}), \mathrm{M}\left(\mathrm{Sx}_{2 \mathrm{n}}, \mathrm{Bz}, \mathrm{a}, \mathrm{t}\right), \mathrm{M}\left(\mathrm{Ax}_{2 \mathrm{n}}, \mathrm{Sx}_{2 \mathrm{n}}, \mathrm{a}, \mathrm{t}\right)\right\}$

Taking the limit $\mathrm{n} \rightarrow \infty$ and using (3) and (4) we have;

$\mathrm{M}(\mathrm{z}, \mathrm{Bz}, \mathrm{a}, \mathrm{kt}) \geq \operatorname{Min}\{1, \mathrm{M}(\mathrm{z}, \mathrm{Bz}, \mathrm{a}, \mathrm{t}), \mathrm{M}(\mathrm{z}, \mathrm{z}, \mathrm{a}, \mathrm{t})\}$

$\mathrm{M}(\mathrm{z}, \mathrm{Bz}, \mathrm{a}, \mathrm{kt}) \geq \mathrm{M}(\mathrm{z}, \mathrm{Bz}, \mathrm{a}, \mathrm{t})$

Now, by lemma (2.7) we obtain that $\mathrm{z}=\mathrm{Bz}$. 
Since $\mathrm{Bz}=\mathrm{Tz}$, therefore $\mathrm{Bz}=\mathrm{Tz}=\mathrm{z}$.

Thus we have; $\mathrm{z}=\mathrm{Az}=\mathrm{Sz}=\mathrm{Bz}=\mathrm{Tz}$.

Hence, $\mathrm{z}$ is a common fixed point of $\mathrm{A}, \mathrm{B}, \mathrm{S}$ and $\mathrm{T}$.

Uniqueness: Let $\mathrm{z}$ and $\mathrm{z}$ ' be two common fixed points of the maps $\mathrm{A}, \mathrm{B}, \mathrm{S}$ and $\mathrm{T}$. Then

$\mathrm{z}=\mathrm{Az}=\mathrm{Sz}=\mathrm{Bz}=\mathrm{Tz}$ and $\mathrm{z}^{\prime}=\mathrm{Az} \mathrm{z}^{\prime}=\mathrm{Sz}^{\prime}=\mathrm{Bz}^{\prime}=\mathrm{Tz}$

Now, by (3.43) we have; (at $\mathrm{x}=\mathrm{z}, \mathrm{y}=\mathrm{z}^{\prime}$ )

$\mathrm{M}(\mathrm{Az}, \mathrm{Bz}, \mathrm{a}, \mathrm{kt}) \geq \operatorname{Min}\left\{\mathrm{M}\left(\mathrm{Bz}^{\prime}, \mathrm{Tz}, \mathrm{a}, \mathrm{t}\right), \mathrm{M}(\mathrm{Sz}, \mathrm{Tz}, \mathrm{a}, \mathrm{t}), \mathrm{M}(\mathrm{Az}, \mathrm{Sz}, \mathrm{a}, \mathrm{t})\right\}$.

$M\left(z, z^{\prime}, a, k t\right) \geq \operatorname{Min}\left\{M\left(z^{\prime}, z^{\prime}, a, t\right), M\left(z, z^{\prime}, a, t\right), M(z, z, a, t)\right\}$.

$\mathrm{M}(\mathrm{z}, \mathrm{z}, \mathrm{a}, \mathrm{kt}) \geq \operatorname{Min}\left\{1, \mathrm{M}\left(\mathrm{z}, \mathrm{z}^{\prime}, \mathrm{a}, \mathrm{t}\right), 1\right\}$

$M\left(z, z^{\prime}, a, k t\right) \geq M\left(z, z^{\prime}, a, t\right)$

Now, by lemma (2.7) we obtain that; $z=z$ '.

Hence; $\mathrm{z}$ is the unique common fixed point of the four self maps $\mathrm{A}, \mathrm{B}, \mathrm{S}$ and T.

This completes the proof.

If we take $\mathrm{B}=\mathrm{A}$ in theorem 3.4 we get the following corollary for three self maps.

Corollary 3.5: Let $\mathrm{A}, \mathrm{S}$ and $\mathrm{T}$ be self mappings of a complete fizzy 2-metric space $(\mathrm{X}, \mathrm{M}, *)$. Suppose that they satisfy the following conditions;

(a) $\mathrm{A}(\mathrm{X}) \subseteq \mathrm{S}(\mathrm{X}) \cap \mathrm{T}(\mathrm{X})$;

(b)The pair (A, S) and (A, T) are weak compatible,

(c) $M(A x, A y, a, k t) \geq \operatorname{Min}\{M(A y, T y, a, t), M(S x, T y, a, t), M(A x, S x, a, t)\}$, for all $\mathrm{x}, \mathrm{y}, \mathrm{a} \in \mathrm{X}, \mathrm{t}>0$ and $0<\mathrm{k}<1$.

Then $\mathrm{S}, \mathrm{T}$ and $\mathrm{A}$ have a unique common fixed point in $\mathrm{X}$.

Proof - The proof is similar to the proof of theorem (3.4).

If we take $\mathrm{S}=\mathrm{T}=\mathrm{I}$, the identity map on $\mathrm{X}$ in corollary 3.5 , then the conditions (a) and (b) are trivially satisfied. Now, taking only one factor in RHS of the contraction condition (c), we obtain the following corollary as follows. 
Corollary 3.6. Let $\mathrm{A}$ be a self map on a complete fuzzy metric space (X, M, *) such that for some $\mathrm{k} \in(0,1), \mathrm{M}(\mathrm{Ax}, \mathrm{Ay}, \mathrm{a}, \mathrm{kt}) \geq \mathrm{M}(\mathrm{x}, \mathrm{y}, \mathrm{a}, \mathrm{t}) \forall \mathrm{x}, \mathrm{y}, \mathrm{a} \in \mathrm{X}, \mathrm{t}>$ 0.Then, A has a unique common fixed point in X.

The following theorem for four self maps in complete fuzzy 3-metric space was proved by Singh Jain and Jain in [18].

Theorem C [18]. Let A, B Sand T be self mappings of a complete fuzzy 3- metric space $(\mathrm{X}, \mathrm{M}, *)$. Suppose that they satisfy the following conditions;

(C.11) $\mathrm{A}(\mathrm{X}) \subseteq \mathrm{T}(\mathrm{X}), \mathrm{B}(\mathrm{X}) \subseteq \mathrm{S}(\mathrm{X})$;

(C.12) A or $\mathrm{S}$ is continuous;

(C.13) The pair (A, S) is compatible and $(\mathrm{B}, \mathrm{T})$ is weak compatible.

(C.14) There exists $\mathrm{k} \in(0,1)$ such that for all $\mathrm{x}, \mathrm{y}, \mathrm{a}, \mathrm{b} \in \mathrm{X}$ and $\mathrm{t}>0$.

$M(A x, B y, a, b, k t) \geq \operatorname{Min}\{M(B y, T y, a, b, t), M(S x, T y, a, b, t), M(A x, S x, a, b$, t) $\}$;

(C.15) $\lim _{\mathrm{t} \rightarrow \infty} \mathrm{M}(\mathrm{x}, \mathrm{y}, \mathrm{z}, \mathrm{w}, \mathrm{t})=1 \forall \mathrm{x}, \mathrm{y}, \mathrm{z}, \mathrm{w} \in \mathrm{X}$ and $\mathrm{t}>0$.

Then A, B, S and T have a unique common fixed point in $\mathrm{X}$.

Now, we prove the following.

Theorem 3.7. Let A, B, S and T be self mappings of a complete fuzzy 3-metric space $(\mathrm{X}, \mathrm{M}, *)$. Suppose that they satisfy the following conditions.

(3.71) $\mathrm{A}(\mathrm{X}) \subseteq \mathrm{T}(\mathrm{X}), \mathrm{B}(\mathrm{X}) \subseteq \mathrm{S}(\mathrm{X})$;

(3.72) the pair (A, S) and (B, T) are weak compatible.

(3.73) There exists $\mathrm{k} \in(0,1)$ suchthat for all $\mathrm{x}, \mathrm{y}, \mathrm{a}, \mathrm{b} \in \mathrm{X}$ and $\mathrm{t}>0$,

$M(A x, B y, a, b, k t) \geq \operatorname{Min}\{M(B y, T y, a, b, t), M(S x, T y, a, b, t), M(A x, S x, a, b$, t) ;

Then A, B, S and T have a unique common fixed point in X.

Proof: The proof is similar to the proof of Theorem (3.4), we hence omit the details. 
If we take $\mathrm{B}=\mathrm{A}$ in theorem (3.7) then we get the following corollary for three self maps.

Corollary 3.8: Let $\mathrm{A}, \mathrm{S}$ and $\mathrm{T}$ be self mappings of a complete fuzzy 3-metric space $(\mathrm{X}, \mathrm{M}, *)$. Suppose that they satisfy the following conditions;

(a) $\mathrm{A}(\mathrm{X}) \subseteq \mathrm{S}(\mathrm{X}) \cap \mathrm{T}(\mathrm{X})$;

(b) The pair (A, S) and (A, T) are weak compatible.

(c) $M(A x, A y, a b, k t) \geq \operatorname{Min}\{M(A y, T y, a, b$ t) $, M(S x, T y, a, b, t), M(A x$, Sx, a, b, t) $\}$, for all $\mathrm{x}, \mathrm{y}, \mathrm{a}, \mathrm{b} \in \mathrm{X}, \mathrm{t}>0$ and $0<\mathrm{k}<1$.

Then $\mathrm{S}, \mathrm{T}$ and $\mathrm{A}$ have a unique common fixed point in $\mathrm{X}$.

Proof. The Proof is similar to the proof of theorem (3.4).

If we take $\mathrm{S}=\mathrm{T}=\mathrm{I}$, the identity map on $\mathrm{X}$ in corollary 3.8 , then the conditions (a) and (b) are trivially satisfied. Now, taking only one factor in RHS of the contraction condition (C), we obtain the, following corollary as follows; Corollary 3.9. Let A be a self map on a complete fuzzy 3- metric space (X, M, *) such that for some $\mathrm{k} \in(0,1)$,

$M(A x, A y, a, b, k t) \geq M(x, y, a, b t)$, for all $x, y, a, b \in X, t>0$.

Then, A has a unique common fixed point in $\mathrm{X}$.

\section{Conclusion}

Our results generalize and improve results of Singh, Jain and Jain in the sense that the compatibility of maps has been replaced by their weak compatibility and the required continuity have been removed that is we have been proved results under weaker conditions. Hence we have been proved more complete results compare to results which have been proved by Singh, Jain and Jain. 


\section{References}

[1] Y.J.Cho: Fixed point in fuzzy metric spaces, journal of fuzzy mathematics 5, 949 - 962(1997).

[2] B.C.Dhage: On common fixed point of coincidentally commuting mappings in D-metric spaces, Indian journal of pure and Applied Mathematics 30,395-406 (1999).

[3] S.Gahler:- 2-metricsche aume ihre topologische structure, Math.Nachr.26 (63-64), 115-148.

[4] A.George and P.Veeramani:- On some results in fuzzy metric spaces, Fuzzy Sets and Systems 64, 395-399 (1994).

[5] M.Grabiec:- Fixed points in fuzzy metric spaces, Fuzzy Sets and Systems 7, 385-389 (1988).

[6] V.Gregori, A.Spena:-On fixed point theorems in fuzzy metric spaces, Fuzzy sets and systems, 125 (2002) 245-252.

[7] G.Jungck:-Compatible mappings and common fixed point, International Journal of Mathematics and Mathematical Sciences 9, 771-779, (1986)

[8] G.Jungck and B.E.Rhoades:- Fixed point for set valued functions without continuity, Indian Journal of pure and Applied Mathematics 29,227-238 (1998).

[9] I.Kramosil and J.Michalek:-Fuzzy metric and statistical metric spaces, Kybernetica 11, 326-334 (1975).

[10] S.N.Mishra and S.L.Singh:- Common fixed point of maps in fuzzy metric spaces, International Journal of mathematics and Mathematical Sciences 17,253-258(1994).

[11] S.Sessa:- On a weak commutative condition in fixed point consideration, Publ.Inst. Math (Beograd) 32,146-153 (1982).

[12] S.Sharma; Common fixed point theorems in fuzzy metric spaces, Fuzzy Sets and Systems, 125(2001), 1-8. 
[13] S.Sharma; Common fixed point theorems in fuzzy metric spaces, Fuzzy Sets and Systems, 127(2002), 345-352.

[14] S.Sharma and B.Deshpande; Discontinuity and Weak compatibility in fixed point consideration on Non-complete fuzzy metric space, The Journal of Fuzzy Mathematics Vol.11, No.3 (2003), 671-686.

[15] B.Singh and Shishir Jain: - Semi compatibility and fixed point theorems in Menger space, Journal of Chingcheong Mathematical Society 17, 1-17 (2004).

[16] B.Singh and Shishir Jain: - A fixed point theorem in menger space through weak compatibility, Journal of Mathematical Analysis and Application 301, 439-448 (2005).

[17] B.Singh and Shishir Jain:- Semi- compatibility, Compatibility and fixed point theorems in fuzzy metric space, Journal of the Chungcheong Mathematical Society 18, 1-22 (2005).

[18] B.Singh, S. Jain and S.Jain; Generalized theorems on fuzzy metric spaces, Southeast Asian Bulletin of Mathematics (2007) 31, 963-978.

[19] G.Song, Comments on "A common fixed point theorem in a fuzzy metric space" Fuzzy sets and systems, 135 (2003) 409-413

[20] Sushil Sharma: - On fuzzy metric spaces, Southeast Asian Bulletin of Mathematics 26, 133-145 (2002).

[21] R.Vasuki:- Common fixed point theorem in a fuzzy metric space, Fuzzy Sets and Systems 97,395-397 (1998).

[22] R.Vasuki:- Common fixed points for R-weakly commuting maps in fuzzy metric space, Indian Journal of Pure and Applied Mathematics, 419-423 (1999).

[23] P.Vijayaraju, M.Marudai, Fixed point theorems for fuzzy mappings, Fuzzy Sets and Systems, 135 (2003) 401-408.

[24] L.A.Zadeh:- Fuzzy sets, Inform and control 89, 338-353 (1965). 